\title{
miR-30e acts as a tumor suppressor in hepatocellular carcinoma partly via JAK1/STAT3 pathway
}

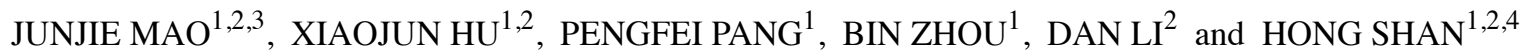 \\ ${ }^{1}$ Center for Interventional Medicine, The Fifth Affiliated Hospital of Sun Yat-sen University; ${ }^{2}$ Guangdong Provincial \\ Engineering Research Center of Molecular Imaging, The Fifth Affiliated Hospital of Sun Yat-sen University, Zhuhai, \\ Guangdong 519000; ${ }^{3}$ Department of Radiology, The Third Affiliated Hospital of Sun Yat-sen University, Guangzhou, \\ Guangdong 510630; ${ }^{4}$ Institute of Interventional Radiology, Sun Yat-sen University, Zhuhai, Guangdong 519000, P.R. China
}

Received August 24, 2016; Accepted January 23, 2017

DOI: $10.3892 /$ or.2017.5683

\begin{abstract}
Hepatocellular carcinoma (HCC) is the leading cause of cancer-associated mortalities. The effective diagnostic and therapeutic targets for HCC are still unclear. miR-30e was differentially expressed in the majority of HCC tissues and cell lines. The aim of this study was to investigate the functional roles of miR-30e and their modulation of cancer networks in HCC cells. We determined the expression of miR-30e by quantitative real-time polymerase chain reaction, and found downregulation of miR-30e in HepG2 and $\mathrm{HuH} 7$ cells. miR-30e mimics significantly inhibited the proliferation, migration, and invasion of HepG2 and $\mathrm{HuH} 7$ cells, and promoted cell apoptosis, but did not influence the cell cycle. Dual-luciferase reporter assays were applied to identify JAK1 as target of miR-30e. miR-30e mimics downregulated the expression levels of JAK1 and vimentin in mRNA and protein in HepG2 and $\mathrm{HuH} 7$ cells. Silence of JAK1 by small interfering RNAs inhibited cell proliferation, migration and invasion of HCC cells. Furthermore, we verified that, IL-6, an agonist of JAK1/STAT3 pathway partly recovered the inhibition of miR-30e mimics on cell migration. Taken together, these findings confirmed our speculation that the functional effect of miR-30e on HCC cells, in part, is dependent on the JAK1/STAT3 signaling pathway. It was suggested that miR-30e has a critical role in the suppression of HCC and presents a novel mechanism of miRNA-mediated JAK1 expression in cancer cells that might be a good prognostic marker for survival of HCC patients.
\end{abstract}

Correspondence to: Professor Hong Shan, Center for Interventional Medicine, Interventional Institution of Sun Yat-sen University, the Fifth Affiliated Hospital of Sun Yat-Sen University, Meihua Road, Zhuhai, Guangdong 519000, P.R. China

E-mail: shanhong@mail.sysu.edu.cn

Key words: HCC, miR-30e, JAK1, vimentin, JAK1/STAT3 pathway

\section{Introduction}

Hepatocellular carcinoma (HCC) is one of the most common malignancies, and is also the leading cause of cancer-associated mortalities $(1,2)$. Although progress has been achieved in the development of new treatment strategies, HCC remains difficult to diagnose at an early stage. Most HCC patients suffer of asymptomatic presentation at the early stage, resulting in metastasis once diagnosis (3). The clinical outcome of advanced HCC patients is still extremely poor. Most advanced HCC patients die as a result of rapid tumor progression, and hepatic resection or transplantation are the only potential curative therapy strategies for patients with HCC (4). However, the effective diagnostic and therapeutic targets are still unclear. Carcinogenesis of HCC is a complex process involving multiple factors, and multi-steps (5). To improve the clinical outcome of HCC therapy, it is critical to elucidate the molecular pathogenesis of HCC and investigate the genes responsible for HCC development and progression.

MicroRNAs (miRNAs) are a group of small noncoding RNAs (19-22 or 19-25 nucleotides) and play an important role in the regulation of gene expression at the posttranscriptional level (6). It was demonstrated that miRNA plays a critical role in the regulation of a variety of physiological and pathological processes, such as development, cell proliferation, cell apoptosis, cell differentiation, cell fate determination, and signal transduction (6-8). To date, miRNAs control nearly $60 \%$ of human genes (7), and more than 1000 human miRNAs have been identified. Increased evidence showed that miRNA act as oncogenes or tumor suppressor genes, dysregulation of them in human malignant tumors regulate the development and progression of cancer via downregulation of their targeted tumor suppressor genes or oncogene expression (9).

Recently, it was demonstrated miR-30e was down-regulated in both plasma and breast cancer tissues (10), non-small cell lung cancer (11), as well as liver tumor tissues (12-14). It was also demonstrated that miR-147a is upregulated in hepatitis $\mathrm{C}$ virus-associated diffuse large B-cell lymphoma, and in small cell lung cancer (15), human gastric cancer (16), squamous cell carcinoma of tongue (17), and hepatocellular carcinoma (18). Some studies demonstrate that miR-147a plays critical effects 
on cell development, migration, and invasion, but has no influence on apoptosis $(19,20)$. In gastric cancer, AKT2 and cyclin D1 were identified as direct targets in gastric cancer, contributing to miR-147 strong inhibitory effect on G1/S transition (20). Hypoxia-induced HIF-1 $\alpha$ increases the expression of miR-147a via HNF4A, miR-147a induced cell proliferation arrest under hypoxia (21). Therefore, each miRNA might target a different gene to play distinct roles in the regulation of fundamental cellular processes.

Herein, we show that miR-30e and miR-147a were differentially expressed in HCC cells (HepG2, MHCC97H, HuH7, and Bel-7402), and the liver cells L02. Two cell lines significantly downregulated the miR-30e expression were selected to investigate the effect of miR-30 on development and progression of HCC, including cell proliferation, cell apoptosis, cell migration and invasion. Mechanistically, we demonstrate that miR-30e target the JAK1-STAT3-vimentin signaling pathway which could collectively contribute to their efficient therapeutic significance, and that IL-6 (agonist of the JAK1/STAT3 pathway) treatment could phenocopy miR-30e downexpression and rescue the cell function induced by miR-30e mimic transfection.

\section{Materials and methods}

Cell culture. The human hepatocellular carcinoma cell lines HepG2, MHCC97H, HuH7, and Bel-7402, and the live cell line L-02, and human embryonic kidney 293 (HEK-293) cells were purchased from Cell Bank of Shanghai Institute of Biochemistry and Cell Biology, Chinese Academy of Sciences. As previous described (22), cells were cultured in RPMI-1640 medium (Hyclone, Logan, UT, USA) and supplemented with $10 \%$ fetal bovine serum (Gibco, Carlsbad, CA, USA), $1 \%$ penicillin/streptomycin at $37^{\circ} \mathrm{C}$ in $5 \% \mathrm{CO}_{2}$.

Cell transfection. The miR-30e mimics, small-interfering RNAs targeting JAK1 (siJAK1) and their respective negative controls were obtained from GenePharma (Shanghai GenePharma Co. Ltd., Shanghai, China). The primers were as follows: miR-30e mimics: 5'- UGU AAA CAU CCU UGA CUG GAAG-3' (forward), and 5'-UGG UGU UAG UUG GUU GCG UUUU-3' (reverse); mimic NC: 5'-UUC UCC GAA CGU GUC ACG UTT-3' (forward), 5'-ACG UGA CACG UUC GGA GAATT-3' (reverse); JAK1 siRNA: 5'-UUG UUU UGU UUU GUU UGA GCC-3' (forward), and 5'-CUC AAA CAA AAC AAA ACA AAA-3' (reverse). Cells were seeded into sixwell plates, incubated for $24 \mathrm{~h}$ before transfected with miR-30e mimics or siJAK1 by using Lipofectamine-2000 (Invitrogen, Carlsbad, CA, USA) in according to the manufacturer's instructions. At $48 \mathrm{~h}$ post-transfection, cells were harvested, expression of miR-30e and JAK1 was tested, and prepared for subsequent experiments.

Luciferase reporter assay. The fragment of wild-type JAK1 3'-UTR (wt 3'-UTR) containing predicted miR-30e binding sites was amplified by PCR, and mutant JAK1 3'-UTR (MUT 3'-UTR) was obtained by mutating the conserved binding sites for miR-30e. The fragments including the wt 3'-UTR or MUT 3'-UTR regions of JAK1 were cloned into XhoI/NotI-digested psiCHECK-2 vector (Promega, Madison, WI, USA), which included both renilla and firefly luciferase reporter genes. Then the psiCHECK-2 vectors with wt 3'-UTR or MUT 3'-UTR regions of JAK1 were transfected into HEK23 cells and transfected with miR-30e mimics or negative control mimics, respectively. After $24 \mathrm{~h}$, the firefly and renilla luciferase activities in cells were determined with a dual-luciferase reporter assay system (Promega) in accordance with the manufacturer's instructions.

Quantitative real-time PCR. Total RNA in transfected cells was isolated using the RNeasy Plus Mini kit (Qiagen) according to the manufacturer's instructions. The expression level of miR-30e was determined using Taqman miRNA assays (Applied Biosystems, Foster City, CA USA) with miRNA-specific primers (forward primer: 5'-ACA CTC CAG CTG GGT GTA AAC ATC CTTG-3' and universal reverse primer: 5'-CTC AAC TGG TGT CGT GGA GTC GGC AAT TCA GTT GAG CTT CCA GTC-3'). For data normalization, U6 small nuclear RNA was used as an endogenous control. The expression level of JAK1 mRNA was determined using PrimeScript RT-PCR kits (Takara, Shiga, Japan) with primers (forward primer: 5'-CGCTCTGGGAAATCTGCT-3' and reverse primer: 5'-TGATGGCTCGGAAGAAAGG-3'), and $\beta$-actin was used as internal control.

Western blotting. Total proteins were extracted from cells as previous described (23) and separated by $10 \%$ SDS-PAGE electrophoresis and then electroblotted onto a nitrocellulose membrane in $25 \mathrm{mM}$ Tris base and $190 \mathrm{mM}$ glycine at $50 \mathrm{~V}$ for $3 \mathrm{~h}$ at $4^{\circ} \mathrm{C}$. The membranes were probed with primary antibodies overnight, followed by incubation with horseradish peroxidase-conjugated secondary antibodies. Protein was detected by enhanced chemiluminescence kit (Amersham Life Science, Buckingham, UK). All antibodies were purchased from Santa Cruz Biotechnology, Santa Cruz, CA, USA.

Cell proliferation assay. Cell proliferation was determined by suing the colorimetric water-soluble tetrazolium salt assay using a Cell Counting Kit-8 (Beyotime, Haimen, China). In brief, cells with a density of $2 \times 10^{3}$ cells per well were seeded in 96-well plates, and then cell proliferation was documented at $24,48,72$, and $96 \mathrm{~h}$. The number of viable cells was obtained by reading the absorbance at $450 \mathrm{~nm}$ using a microplate reader Thermo Plate (Rayto Life and Analytical Science C. Ltd., Wetzlar, Germany).

Cell apoptosis assay. Cell apoptosis was assessed by using an Annexin V-FITC apoptosis detection kit (BD Pharmingen, Franklin Lakes, NJ, USA). In brief, Annexin V-FITC $(5 \mu \mathrm{l})$ and propidium iodide $(5 \mu \mathrm{l})$ were added in $100 \mu \mathrm{l}$ of cells at concentration of $1 \times 10^{6}$ cells $/ \mathrm{ml}$ and incubated in the dark for $15 \mathrm{~min}$. Then, binding buffer was added and apoptosis was analyzed by flow cytometry (BD Biosciences, Franklin Lakes, NJ, USA).

Cell cycle assay. After transfection, cells were harvested after trypsinization and were resuspended with concentration of $1 \times 10^{6}$ cells $/ \mathrm{ml}$ and prepared using Cycle Test Plus DNA Reagent kit (Becton Dickinson, San Jose, CA, USA) 

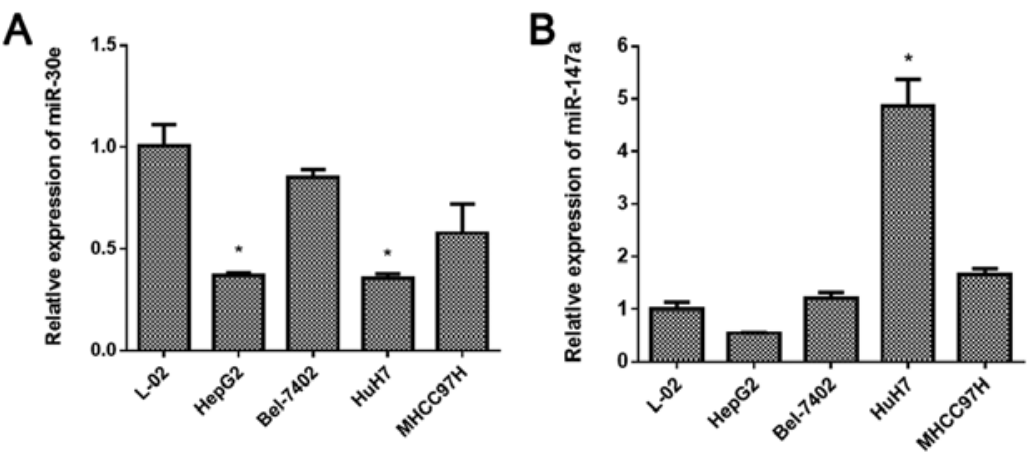

C

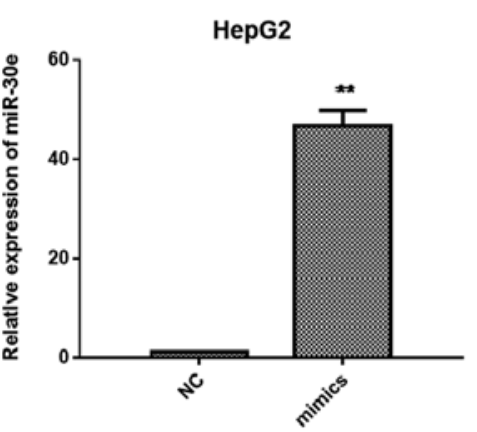

D

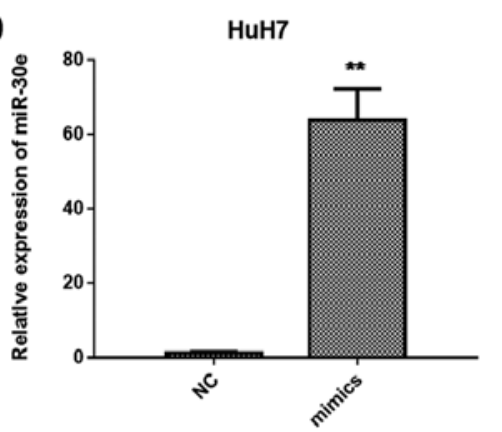

HepG2
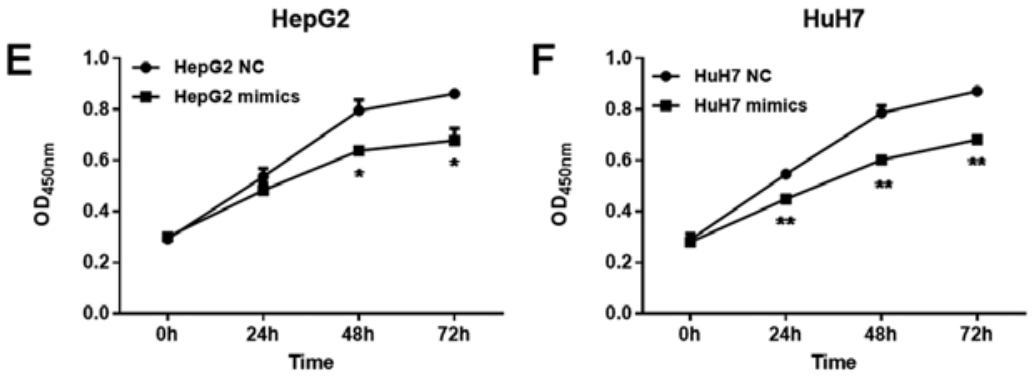
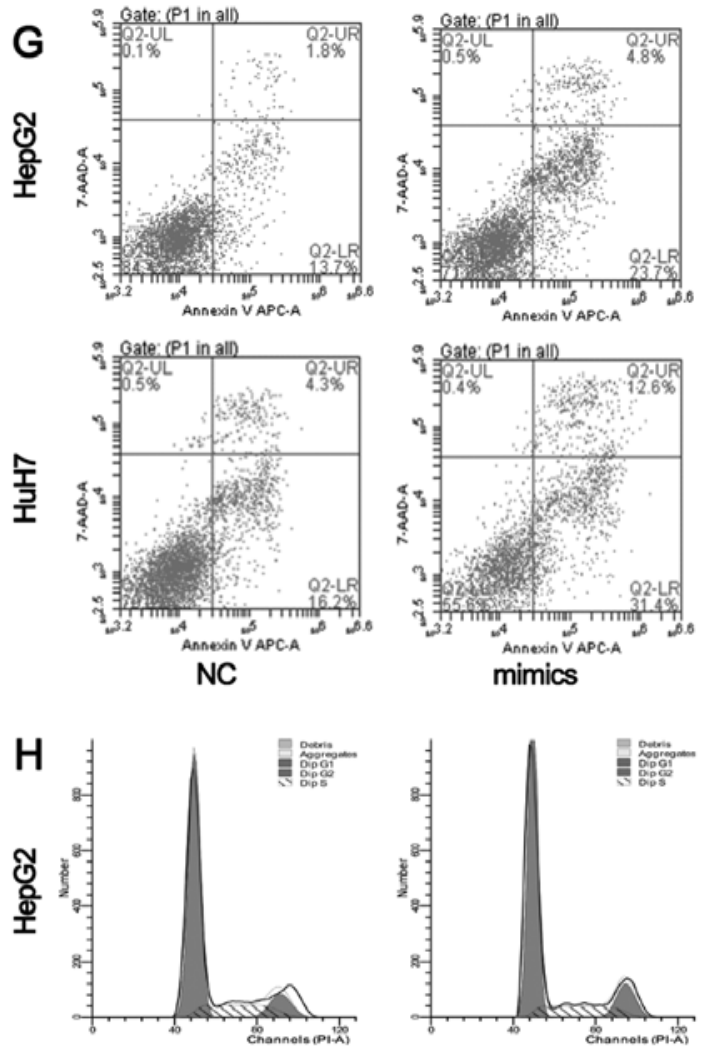

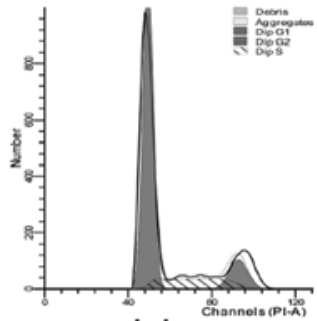

mimics

Figure 1. Effect of miR-30e on HCC cell. Expression of (A) miR-30e, (B) miR-147a in HepG2, MHCC97H, HuH7, and Bel-7402 hepatoma carcinoma cell lines, and L-02 live cell line. ${ }^{*} \mathrm{P}<0.05$ vs. L-02. miR-30e was overexpressed in mimic transfected HepG2 (C) and HuH7 (D) cells. ${ }^{*} \mathrm{P}<0.05$, ${ }^{* *} \mathrm{P}<0.01$ vs. NC. (E and F) CCK-8 assays; (G) cell apoptosis; $(\mathrm{H})$ cell cycle.

according to the manufacturer's instructions. Cell cycle was analyzed by flow cytometer using propidium iodide (PI) as a specific fluorescent dye probe. The PI fluorescence intensity of 10,000 cells was measured for each sample using a BectonDickinson FACSCalibur flow cytometer.

Migration and invasion. Cell migration and invasion of HCC cells (HepG2 and $\mathrm{HuH7}$ cells) were performed by using a Transwell chamber (Millipore, Billerica, MA, USA). For cell invasion, transwell chamber was coated with $30 \mu$ l Matrigel. Cells were seeded into 24 -well plate and cultured at $37^{\circ} \mathrm{C}$ in RPMI-1640 medium with $2 \%$ serum, while $600 \mu \mathrm{l}$ of $10 \%$ FBS RPMI-1640 was added to the lower chamber. After $48 \mathrm{~h}$, HCC cells were fixed with $100 \%$ methanol for $30 \mathrm{~min}$ and stained using crystal violet for $20 \mathrm{~min}$. Non-migrated cells were removed using cotton swabs. Cell images were obtained under a phase-contrast microscope (Olympus, Tokyo, Japan).

Statistical analysis. The results are expressed as the mean \pm SD. Statistical evaluation was performed using GraphPad
Prism software version 5.01 (GraphPad, Inc., La Jolla, CA, USA). The normal distribution of variables was assessed prior to selecting the tests to use for statistical analyses with ANOVA or student t-test. A P-value $<0.05$ was considered significant.

\section{Results}

miR-30e is significantly downregulated in HepG2 and $\mathrm{HuH7}$ cells, whereas miR-147a is significantly upregulated in $\mathrm{HuH7}$ cells. According to the miRNA array analysis data $(24,25)$, we tested the expression of miR-30e, miR-147a in HepG2, MHCC97H, HuH7, and Bel-7402 hepatoma carcinoma cell lines, and L-02 live cell line to screen the most pronounced miRNA by qRT-PCR (Fig. 1A and B). miR-30e was significantly downregulated in both HepG2 and $\mathrm{HuH} 7$ cells, while it was not significantly changed in L-02, Bel-7402, and MHCC97H cells. miR-147a was significantly upregulated in $\mathrm{HuH} 7$ cells, but not in other cell lines. Thus, we further examined the role of miR-30e in HepG2 and HuH7 cell lines. 

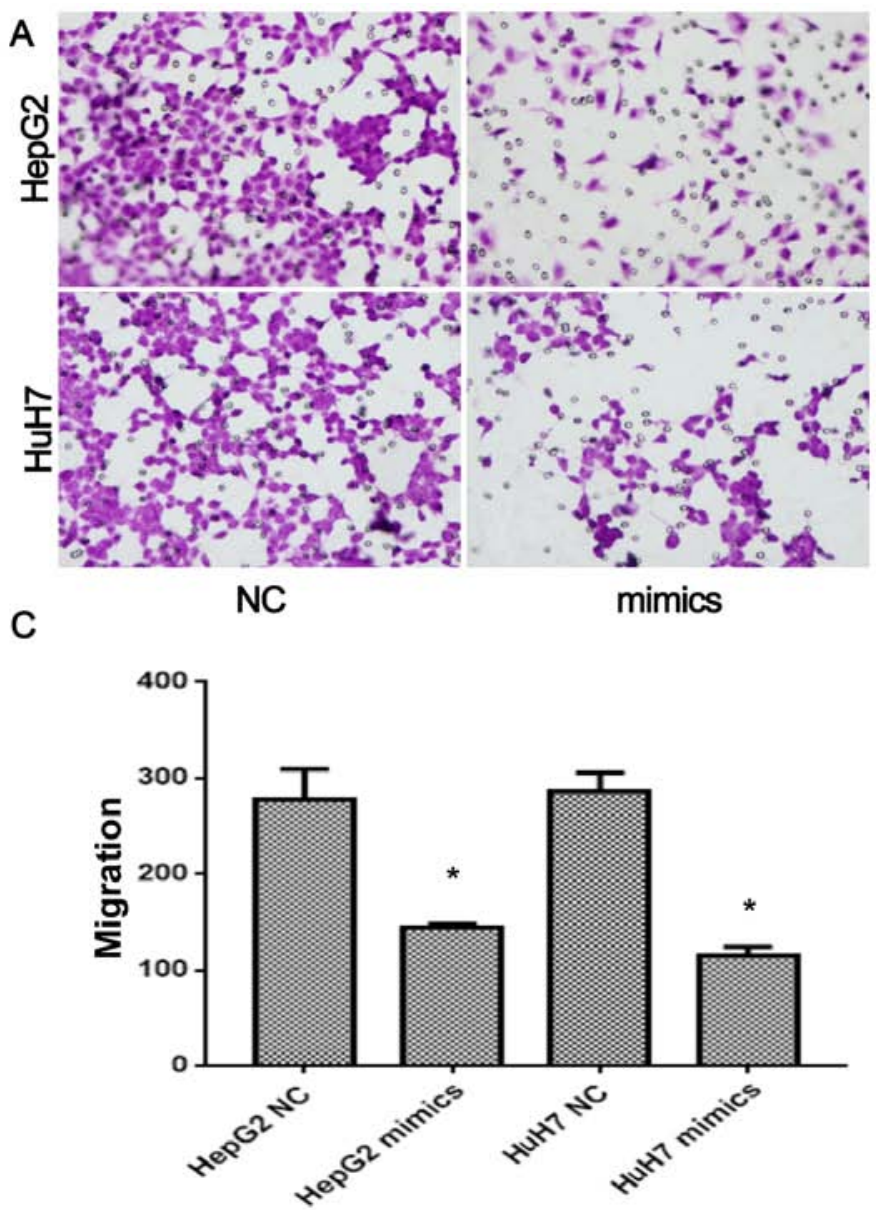
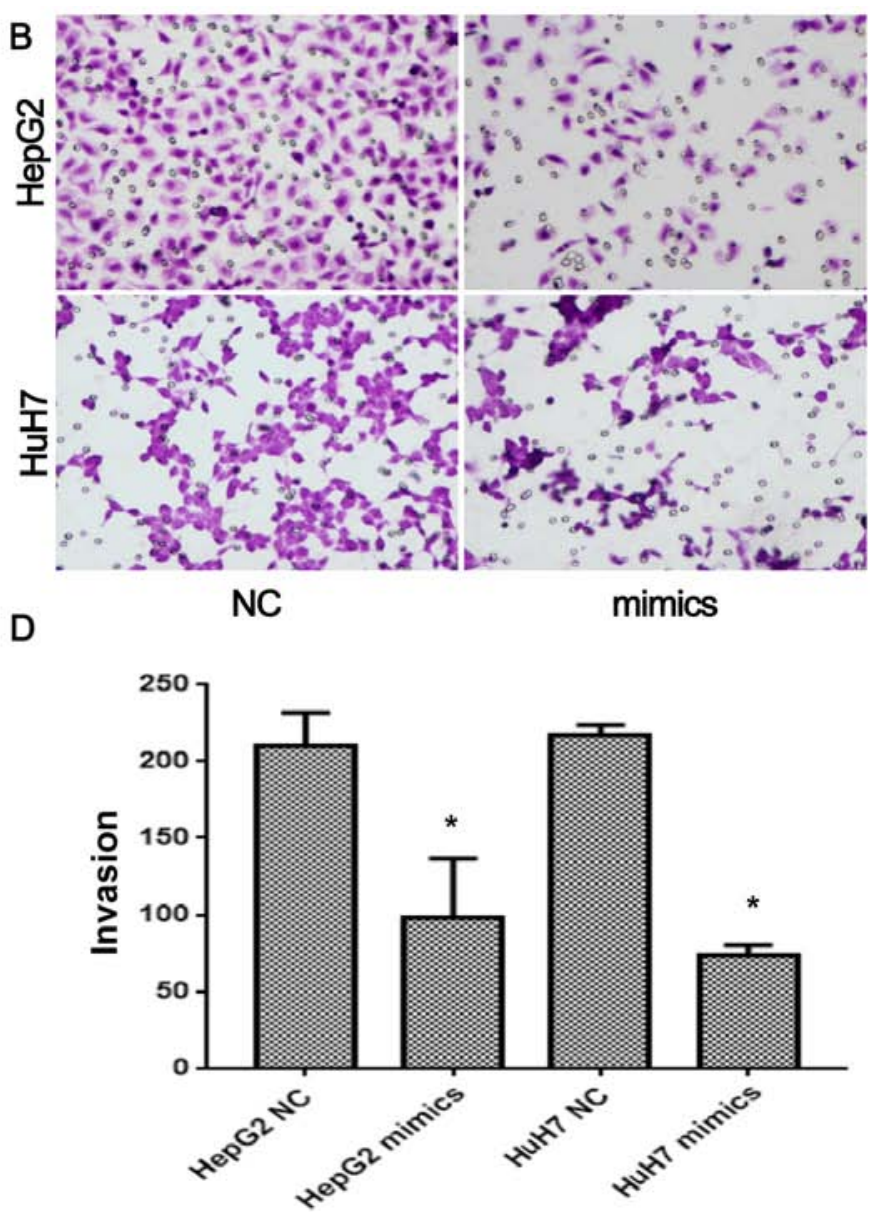

Figure 2. miR-30e mimics inhibited the cell migration and invasion of HCC cells. (A) Migration; (B) invasion; (C) quantification of migration; (D) quantification of invasion. ${ }^{*} \mathrm{P}<0.05$, vs. NC.

miR-30e mimics inhibit the proliferation, migration, and invasion of HepG2 and HuH7 cells, and promote cell apoptosis, but do not influence the cell cycle. After transfection of miR-30e mimics, miR-30e was overexpressed in HepG2 cells $(\mathrm{P}<0.01$; Fig. 1C) and HuH7 cells $(\mathrm{P}<0.01$; Fig. 1D). The proliferation of HepG2 was significantly inhibited by miR-30e mimics after $48 \mathrm{~h}(\mathrm{P}<0.05$; Fig. 1E). Similar change in proliferation was observed in $\mathrm{HuH} 7$ cells. After $24 \mathrm{~h}$, the proliferation of $\mathrm{HuH7}$ cells was significantly inhibited by miR-30e mimics $(\mathrm{P}<0.05,0.01$ at $24,48,72 \mathrm{~h}$, respectively; Fig. 1F). Furthermore, miR-30e mimics promoted apoptosis of HepG2 and HuH7 cells (Fig. 1G). However, miR-30e mimics did not significantly change the cell cycle (Fig. $1 \mathrm{H})$. The migration and invasion of HepG2 and $\mathrm{HuH} 7$ cells after transfection of miR-30e were also detected (Fig. 2A-D). miR-30e mimics significantly inhibited the migration of HepG2 and HuH7 cells ( $\mathrm{P}<0.05$, Fig. 2A and C). Similarly, the invasion of HepG2 and $\mathrm{HuH} 7$ cells after transfection of miR-30e were also significantly inhibited $(\mathrm{P}<0.05$, Fig. 2B and D). Thus, overexpression of miR-30e significantly inhibited the proliferation, migration, and invasion of HCC cells, and promoted cell apoptosis.

$J A K 1$, not vimentin is a direct target of $m i R-30 e$. Luciferase reporter assay was used to validate the target of miR-30e (Fig. 3A-D). The Reporter luciferase vectors, wt-3'UTR of
JAK1, vimentin containing the predicted miR-30e binding sites and the corresponding mutated vectors (Mut-3'UTR) were achieved (Fig. 3A and B) and transfected into HepG2 and $\mathrm{HuH7}$ cells. The luciferase activity was inhibited by miR-30e in the cells with wt-3'UTR of JAK1 (P<0.05, Fig. 3C), but not changed in mut-3'UTR of JAK1, or wt-and Mut-3'UTR of vimentin (Fig. 3D), suggesting JAK1, not vimentin is a direct target of miR-30e. Furthermore, the expression of JAK1 and vimentin in miR-30e mimics transfected-HepG2 and $\mathrm{HuH} 7$ cells were performed by qRT-PCR and western blotting (Fig. 3C-H). miR-30e mimics inhibited the expression of JAK1 and vimentin in mRNA and protein levels in HepG2 and HuH7 cells $(\mathrm{P}<0.05$, Fig. 3E and F). Thus, overexpression of miR-30e significantly downregulated the expression of JAK1 and vimentin, but only JAK1 was a direct target of miR-30e.

Silence of JAK1 inhibits cell proliferation, migration and invasion of HCC cells. To further confirm whether miR-30e effects HCC cells via JAK1, JAK1 siRNA (siJAK1) was transfected in the cells, and the expression of JAK1 in mRNA and protein levels was detected by qRT-PCR (Fig. 4A) and western blotting (Fig. 4B), respectively. Silence of JAK1 by JAK1 siRNA was confirmed by the lower expression levels of JAK1 in both HepG2 and HuH7 cells $(\mathrm{P}<0.05$; Fig. 4A and $\mathrm{B})$, compared with the non-transfected controls. siJAK1 
A

JAK1 1295: 5'-ACUCUAUATGCACUUUGUUUACU-3' WT UTR miR-30e : 3'- GAAGGUCAGUUCCUACAAAUGU-5' JAK1 1295: 5'- ACTCTATATGCACTTTGTTTACTCT-3' MUT UTR

C

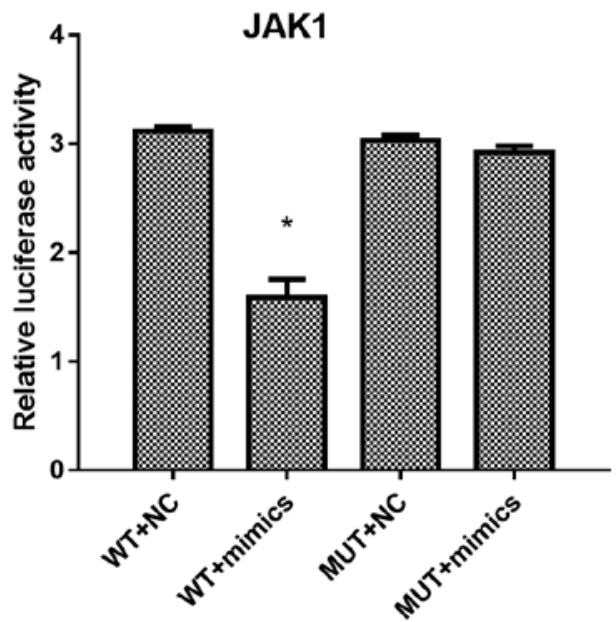

E

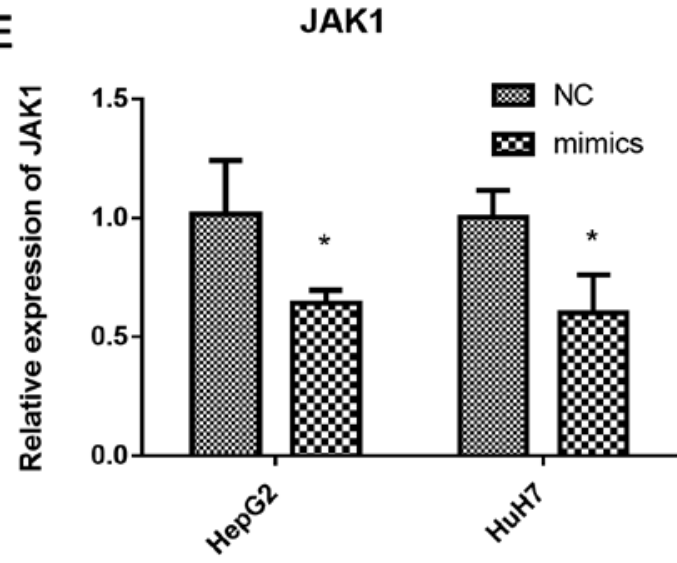

G

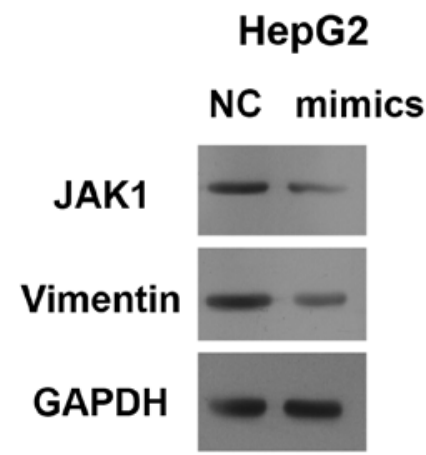

B

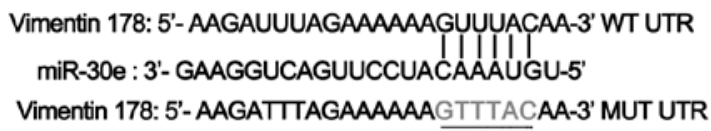

D

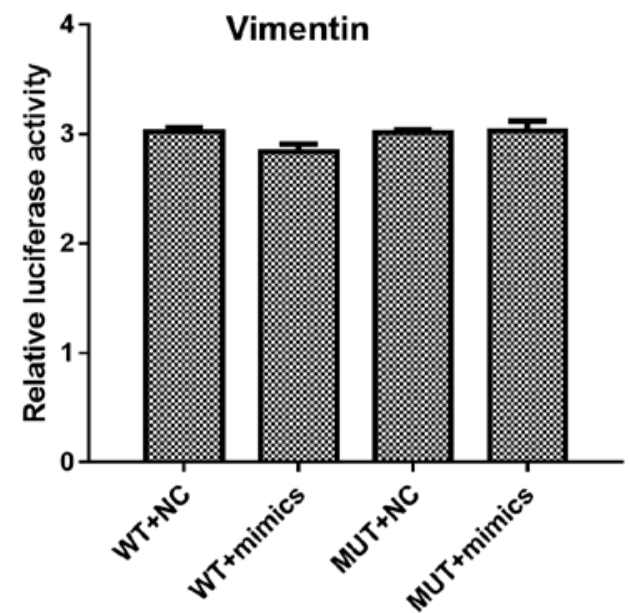

F

$\mathrm{H}$

$\mathrm{HuH} 7$

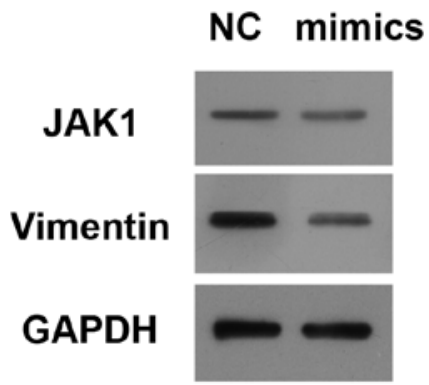

Figure 3. JAK1 acts as a direct target of miR-30e in HCC cells. (A and B) predicted binding sites. (C and D) Luciferase reporter assay. (E and F) mRNA expression of JAK1 and vimentin, respectively. (G and H) Expression of JAK1 and vimentin in protein level, respectively. ${ }^{*} \mathrm{P}<0.05$, vs. WT+NC or NC.

significantly inhibited the proliferation of HepG2 $(\mathrm{P}<0.05$ Fig. 4C) and $\mathrm{HuH} 7(\mathrm{P}<0.05$; Fig. 4D) cells after 48 h. The migration and invasion of the siJAK1-transfected HCC cells were also detected. After siJAK1 transfection, the migration of HepG2 $(\mathrm{P}<0.05$; Fig. 4E and $\mathrm{G})$ and HuH7 cells $(\mathrm{P}<0.05$; Fig. $4 \mathrm{~F}$ and $\mathrm{H}$ ) were significantly inhibited. Similarly, the invasion of HepG2 ( $\mathrm{P}<0.05$; Fig. $4 \mathrm{E}$ and $\mathrm{G})$ and $\mathrm{HuH} 7$ cells $(\mathrm{P}<0.05$; Fig. $4 \mathrm{~F}$ and $\mathrm{H})$ were also inhibited by siJAK1, respectively. These results suggested that miR-30e inhibited cell proliferation, migration, and invasion of HCC cells via direct downregulation of JAK1.

$J A K / S T A K$ pathway mediates the effects of miR-30e. It was demonstrated that vimentin is related to the motility capacity of HCC cells $(5,26)$ and important molecule in JAK/STAK/ vimentin signaling pathway (27). We further investigated 


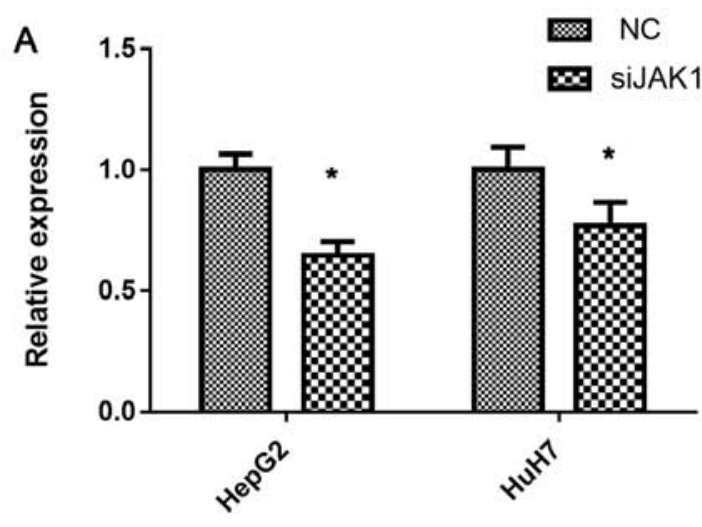

C
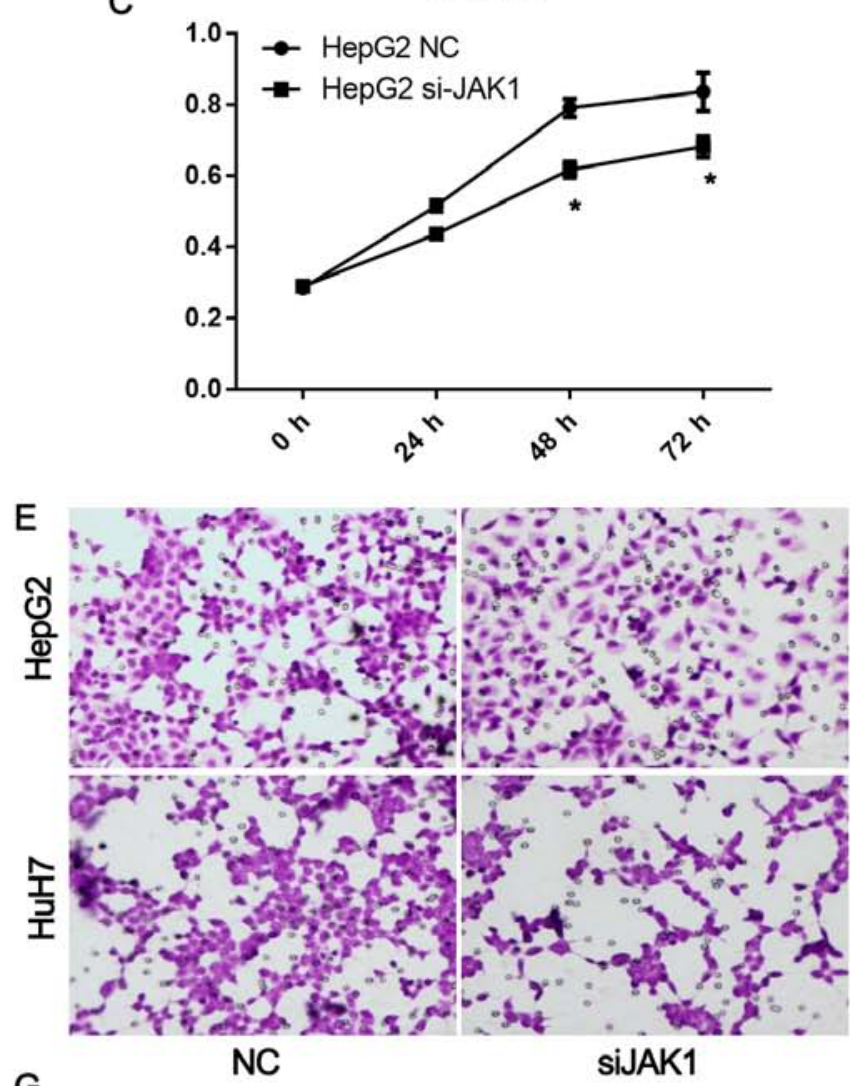

G

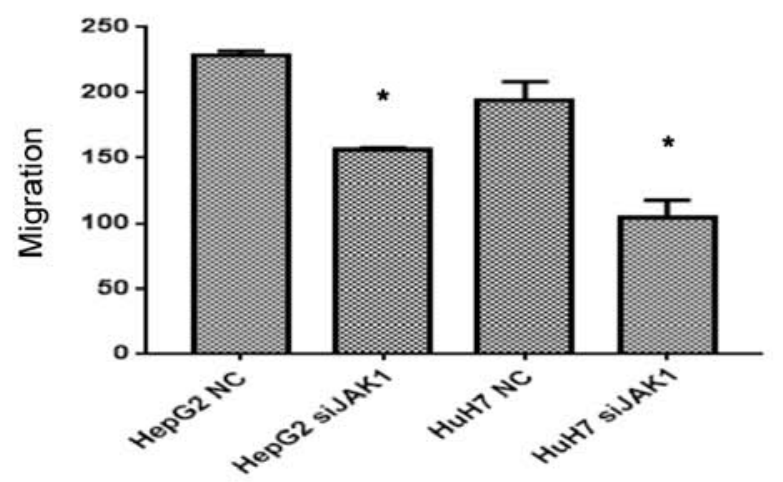

B

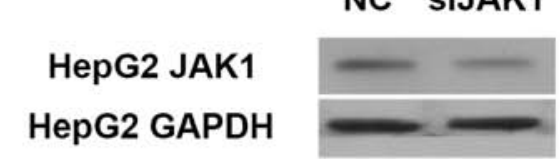

NC SIJAK1

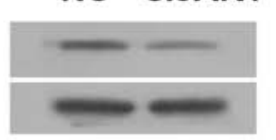

D

HuH7

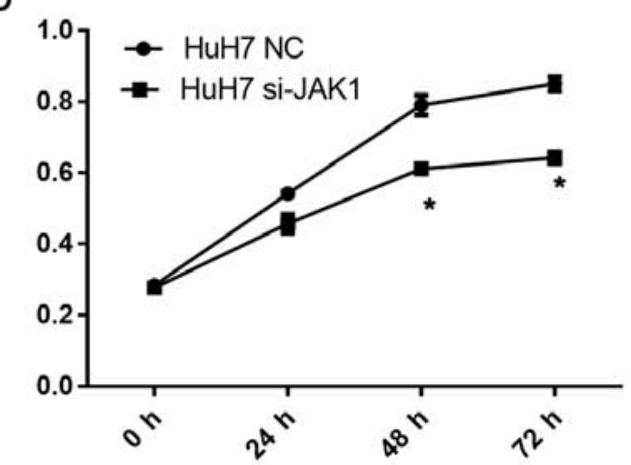

$\mathrm{H}$
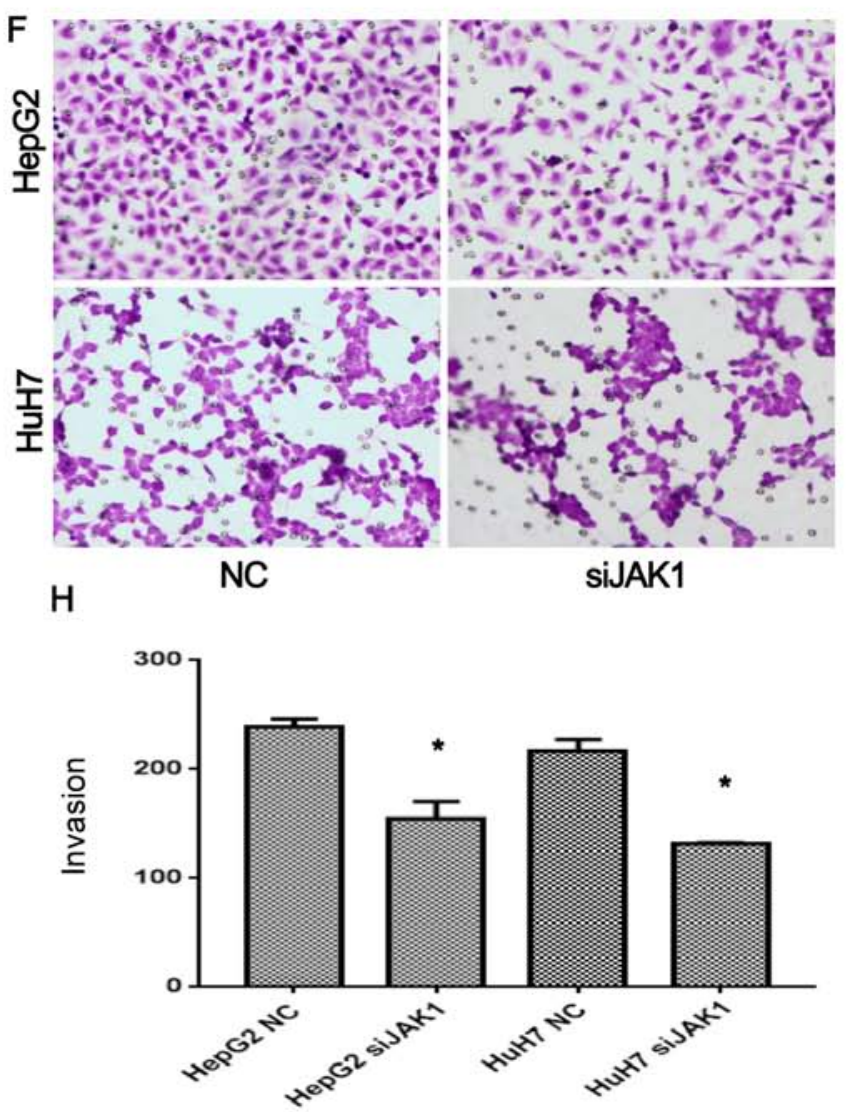

Figure 4. siJAK1 inhibits cell proliferation, migration and invasion of HCC cells. (A and B) validation of the silence of JAK1 by qRT-PCR and western blotting. (C and D) cell proliferation in HepG2 and HuH7 cell lines. (E) Migration; (F) invasion; (G) quantification of migration; (H) quantification of invasion. ${ }^{*} \mathrm{P}<0.05$, vs. NC.

whether JAK/STAK pathway mediated the effect of miR-30e (Fig. 5). First, miR-30e mimics not only signifi- cantly downregulated the expression of JAK1 (Fig. 3G and H) and STAT3 (Fig. 5A), but also significantly downregu- 
A

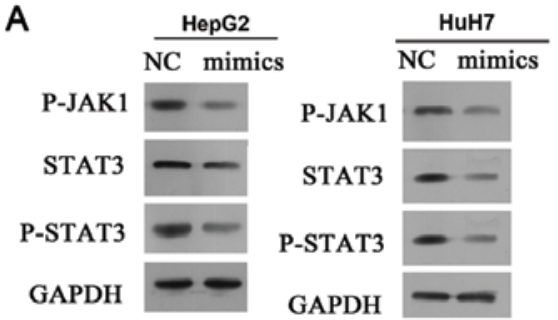

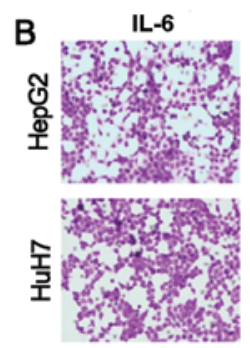
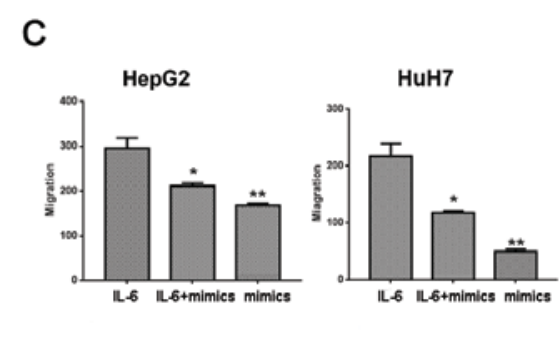

D
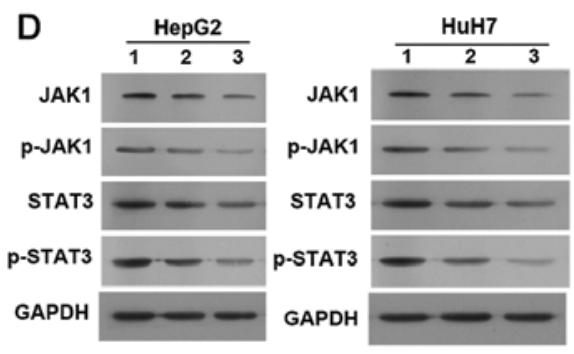

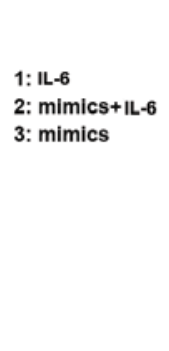

E

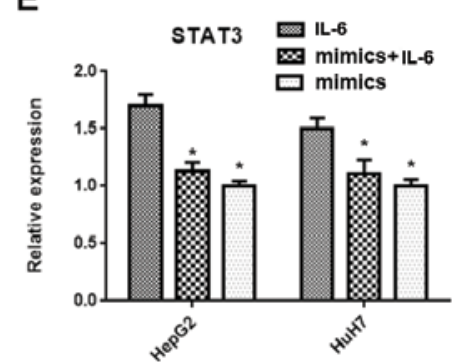

F

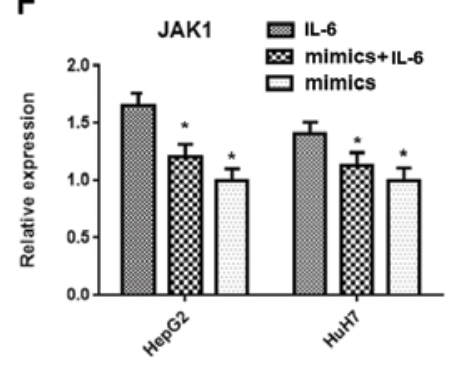

Figure 5. JAK/STAK pathway mediates the effects of miR-30e. (A) Effect of miR-30e on expression and phosphorylation levels of JAK1 and STAT3. (B and C) Migration of cells in the presence of IL-6 and/or mimics. (D) Expression and phosphorylation levels of JAK1 and STAT3 in the presence of IL-6 and/or mimics. (E and F) mRNA expression of STAT3 and JAK1 in the presence of IL-6 and/or mimics. ${ }^{*} \mathrm{P}<0.05,{ }^{* *} \mathrm{P}<0.01$ vs. IL-6.

lated phosphorylation levels of JAK1 and STAT3 (Fig. 5A). Second, IL-6, the agonist of JAK/STAT3 pathway decreased the inhibition of cell migration of HepG2 and HuH7 cells by miR-30e partly $(\mathrm{P}<0.05$, Fig. 5B and $\mathrm{C})$. Third, IL-6 reduced the inhibition of expression (in both $\mathrm{mRNA}$ and protein levels) and phosphorylation levels of JAK1 and STAT3 (Fig. 5D-F). Thus, JAK/STAT3 pathway mediated the effect of miR-30e on cell migration.

\section{Discussion}

Hepatocellular carcinoma (HCC) ranks among the top 10 of the most common malignancies in the world, and is also the leading cause of cancer-associated mortalities in China $(1,2)$. HCC remains difficult to diagnose at an early stage, and hepatic resection or transplantation are the only potential curative therapy strategies for patients with HCC (4). HCC patients with poor diagnosis undergo metastasis fast and die as a result of the rapid tumor progression. To improve the clinical outcome of HCC therapy, it is critical to elucidate the molecular pathogenesis of HCC and investigate the genes responsible for HCC development and progression. Herein, we demonstrate that miR-30e and miR-147a are differentially expressed in HCC cells (HepG2, MHCC97H, HuH7, and Bel-7402), and liver cells L02. miR-30e mimics inhibited the development and progression of HCC including inhibited the cell proliferation, cell migration and invasion, promoted cell apoptosis. Mechanistically, we demonstrate that miR-30e target the JAK1-STAT3-vimentin signaling pathway which could collectively contribute to their efficiently therapeutic significance, and that IL-6 (agonists of the JAK1/STAT3 pathway) treatment could phenocopy miR-30e downexpression and rescue the cell function induced by miR-30e mimics transfection.
Recently, it was demonstrated miR-30e was downregulated in both plasma and breast cancer tissues (10), in non-small cell lung cancer (11), as well as in liver tumor tissues in hepatocellular carcinoma (12-14). It was also demonstrated that miR-147a was upregulated in hepatitis $\mathrm{C}$ virus-associated diffuse large B-cell lymphoma, and in small cell lung cancer (15), human gastric cancer (16), squamous cell carcinoma of tongue (17), and hepatocellular carcinoma (18). Consistently, our results demonstrated the downregulation of miR-30e in HepG2 and HuH7 HCC cell lines, and upregulation of miR-147a only in $\mathrm{HuH} 7$ cell lines. Thus, the HepG2 and HuH7 cell lines were selected for investigation of the miR-30e role.

miR-30e was proved to be suppressor of human NK cell cytotoxicity, and could directly target perforin (28). In breast cancer, it upregulated three predicted targets of miR-30e including RAB11A, BNIP3L, and RAB32 associated with downregulation of miR-30e (10). In addition, miR-30e targeted 3'-untranslated region (3'UTR) of prolyl 4-hydroxylase subunit alpha-1 (P4HA1) mRNA, and reduced the expression of P4HA1 at the levels of mRNA and protein (12). Overexpression of miR-30e suppressed cell proliferation of HepG2 cells and reduced colony formation (12). Herein, we found JAK1 was also the target of miR-30e by luciferase reporter gene assays. Enforced expression of miR-30e inhibited cell proliferation, cell migration and invasion, promoted cell apoptosis, but had no effect on the cell cycle arrest. Silence of JAK1 also inhibited cell proliferation, cell migration and invasion, suggesting miR-30e inhibited the cell proliferation, cell migration and invasion partly via JAK1. miR-23a suppressed the JAK1/STAT-6 pathway and reduced production of M2 type cytokines by targeting JAK1 and STAT-6 directly (29). In acute erythroid leukemia, miR-23a, miR-27a and miR-24 formed a miRNA cluster, synergistically 
targeting multiple members of the oncogenic JAK1-STAT3 pathway, and thus reinforced their inhibition on the cascade to regulate cell proliferation and apoptosis (30). Exogenous miR-9 activated JAK-STAT pathway in tumor angiogenesis (31). MiR-30c also targets JAK1 playing important roles in porcine reproductive and respiratory syndrome virus (32). Therefore, there is a complex network between miRNAs and their targets. The investigation on the correlation between miRNAs and their targets should be helpful in clearing the mechanism.

miR-30e has no effect on the cell cycle. Some studies demonstrate that miR-147a plays critical effects on cell development, migration, and invasion, but has no influence on apoptosis $(19,20)$. In gastric cancer, AKT2 and cyclin D1 were identified as direct targets in gastric cancer, contributing to miR-147 strong inhibitory effect on G1/S transition (20). Hypoxia-induced HIF-1 $\alpha$ increases the expression of miR-147a via HNF4A, miR-147a induced cell proliferation arrest under hypoxia (21). Therefore, each miRNAs might target a different gene to play distinct roles in the regulation of fundamental cellular processes like development and proliferation, cell fate determination and apoptosis.

In conclusion, we demonstrated that miR-30e targets the JAK1-STAT3-vimentin signaling pathway playing critical roles in inhibition of the cascade to regulate cell proliferation and apoptosis, which could collectively contribute to their efficient therapeutic significance.

\section{Acknowledgements}

This work is supported by the National Natural Science Foundation Key Project of China (grant no. 81430041), the National Natural Science Foundation of China (grant nos. 81271621, 81501561, 81620108017), Natural Science Foundation of Guangdong Province (2014A030310043), the Science and Technology Planning Project of Guangzhou Province (201604020098, 201610010006).

\section{References}

1. Ferlay J, Soerjomataram I, Dikshit R, Eser S, Mathers C, Rebelo M, Parkin DM, Forman D and Bray F: Cancer incidence and mortality worldwide: Sources, methods and major patterns in GLOBOCAN 2012. Int J Cancer 136: E359-E386, 2015.

2. Torre LA, Bray F, Siegel RL, Ferlay J, Lortet-Tieulent J and Jemal A: Global cancer statistics, 2012. CA Cancer J Clin 65: 87-108, 2015

3. Yegin EG, Oymaci E, Karatay E and Coker A: Progress in surgical and nonsurgical approaches for hepatocellular carcinoma treatment. Hepatobiliary Pancreat Dis Int 15: 234-256, 2016.

4. Buendia MA and Neuveut C: Hepatocellular carcinoma. Cold Spring Harb Perspect Med 5: a021444, 2015.

5. Zeng YE, Yao XH, Yan ZP, Liu JX and Liu XH: Potential signaling pathway involved in sphingosine-1-phosphate-induced epithelial-mesenchymal transition in cancer. Oncol Lett 12: 379-382, 2016

6. Kim VN: MicroRNA biogenesis: Coordinated cropping and dicing. Nat Rev Mol Cell Biol 6: 376-385, 2005.

7. Lages E, Ipas H, Guttin A, Nesr H, Berger F and Issartel JP: MicroRNAs: Molecular features and role in cancer. Front Biosci (Landmark Ed) 17: 2508-2540, 2012.

8. Bartel DP: MicroRNAs: Genomics, biogenesis, mechanism, and function. Cell 116: 281-297, 2004.

9. Croce CM: Causes and consequences of microRNA dysregulation in cancer. Nat Rev Genet 10: 704-714, 2009.
10. Lin Z, Li JW, Wang Y, Chen T, Ren N, Yang L, Xu W, He H, Jiang Y, Chen X, et al: Abnormal miRNA-30e expression is associated with breast cancer progression. Clin Lab 62: 121-128, 2016.

11. Wang Y, Chen J, Lin Z, Cao J, Huang H, Jiang Y, He H, Yang L, Ren N and Liu G: Role of deregulated microRNAs in non-small cell lung cancer progression using fresh-frozen and formalinfixed, paraffin-embedded samples. Oncol Lett 11: 801-808, 2016.

12. Feng G, Shi H, Li J, Yang Z, Fang R, Ye L, Zhang W and Zhang X: MiR-30e suppresses proliferation of hepatoma cells via targeting prolyl 4-hydroxylase subunit alpha-1 (P4HA1) mRNA. Biochem Biophys Res Commun 472: 516-522, 2016.

13. Wong CM, Wong CC, Lee JM, Fan DN, Au SL and Ng IO: Sequential alterations of microRNA expression in hepatocellular carcinoma development and venous metastasis. Hepatology 55: 1453-1461, 2012.

14. Bhattacharya S, Steele R, Shrivastava S, Chakraborty S, Di Bisceglie AM and Ray RB: Serum miR-30e and miR-223 as novel noninvasive biomarkers for hepatocellular carcinoma. Am J Pathol 186: 242-247, 2016.

15. Yi Z, Fu Y, Ji R, Li R and Guan Z: Altered microRNA signatures in sputum of patients with active pulmonary tuberculosis. PLoS One 7: e43184, 2012.

16. Yao Y, Suo AL, Li ZF, Liu LY, Tian T, Ni L, Zhang WG, Nan KJ, Song TS and Huang C: MicroRNA profiling of human gastric cancer. Mol Med Rep 2: 963-970, 2009.

17. Wong TS, Liu XB, Wong BY, Ng RW, Yuen AP and Wei WI: Mature miR-184 as potential oncogenic microRNA of squamous cell carcinoma of tongue. Clin Cancer Res 14: 2588-2592, 2008.

18. Han ZB, Zhong L, Teng MJ, Fan JW, Tang HM, Wu JY, Chen HY, Wang ZW, Qiu GQ and Peng ZH: Identification of recurrencerelated microRNAs in hepatocellular carcinoma following liver transplantation. Mol Oncol 6: 445-457, 2012.

19. Bertero T, Grosso S, Robbe-Sermesant K, Lebrigand K, Hénaoui IS, Puisségur MP, Fourre S, Zaragosi LE, Mazure NM, Ponzio G, et al: 'Seed-Milarity' confers to hsa-miR-210 and hsa-miR-147b similar functional activity. PLoS One 7: e44919, 2012.

20. Uhlmann S, Mannsperger H, Zhang JD, Horvat EÁ, Schmidt C, Küblbeck M, Henjes F, Ward A, Tschulena U, Zweig K, et al: Global microRNA level regulation of EGFR-driven cell-cycle protein network in breast cancer. Mol Syst Biol 8: 570, 2012.

21. Wang F, Zhang H, Xu N, Huang N, Tian C, Ye A, Hu G, He J and Zhang Y: A novel hypoxia-induced miR-147a regulates cell proliferation through a positive feedback loop of stabilizing HIF-1 $\alpha$. Cancer Biol Ther 17: 790-798, 2016.

22. Zheng L, Deng CL, Wang L, Huang XB, You N, Tang YC, Wu K, Liang P, Mi N and Li J: COMMD7 is correlated with a novel NF-kB positive feedback loop in hepatocellular carcinoma. Oncotarget 7: 32774-32784, 2016.

23. Zeng Y, Sun HR, Yu C, Lai Y, Liu XJ, Wu J, Chen HQ and Liu XH: CXCR1 and CXCR2 are novel mechano-sensors mediating laminar shear stress-induced endothelial cell migration. Cytokine 53: 42-51, 2011.

24. Gramantieri L, Fornari F, Callegari E, Sabbioni S, Lanza G, Croce CM, Bolondi L and Negrini M: MicroRNA involvement in hepatocellular carcinoma. J Cell Mol Med 12: 2189-2204, 2008.

25. Budhu A, Jia HL, Forgues M, Liu CG, Goldstein D, Lam A, Zanetti KA, Ye QH, Qin LX, Croce CM, et al: Identification of metastasis-related microRNAs in hepatocellular carcinoma. Hepatology 47: 897-907, 2008.

26. Lin $X$, Yang Z, Zhang P, Liu Y and Shao G: miR-154 inhibits migration and invasion of human non-small cell lung cancer by targeting ZEB2. Oncol Lett 12: 301-306, 2016.

27. Barcelona PF, Ortiz SG, Chiabrando GA and Sánchez MC: Alpha2-macroglobulin induces glial fibrillary acidic protein expression mediated by low-density lipoprotein receptor-related protein 1 in Müller cells. Invest Ophthalmol Vis Sci 52: 778-786, 2011.

28. Wang P, Gu Y, Zhang Q, Han Y, Hou J, Lin L, Wu C, Bao Y, Su X, Jiang $\mathrm{M}$, et al: Identification of resting and type I IFN-activated human NK cell miRNomes reveals microRNA-378 and microRNA-30e as negative regulators of NK cell cytotoxicity. J Immunol 189: 211-221, 2012.

29. Ma S, Liu M, Xu Z, Li Y, Guo H, Ge Y, Liu Y, Zheng D and Shi J: A double feedback loop mediated by microRNA-23a/27a/24-2 regulates M1 versus M2 macrophage polarization and thus regulates cancer progression. Oncotarget 7: 13502-13519, 2016. 
30. Su R, Dong L, Zou D, Zhao H, Ren Y, Li F, Yi P, Li L, Zhu Y, Ma Y, et al: microRNA-23a, -27a and -24 synergistically regulate JAK1/Stat 3 cascade and serve as novel therapeutic targets in human acute erythroid leukemia. Oncogene 35: 6001-6014, 2016.

31. Zhuang G, Wu X, Jiang Z, Kasman I, Yao J, Guan Y, Oeh J, Modrusan Z, Bais C, Sampath D, et al: Tumour-secreted miR-9 promotes endothelial cell migration and angiogenesis by activating the JAK-STAT pathway. EMBO J 31: 3513-3523, 2012.

32. Zhang Q, Huang C, Yang Q, Gao L, Liu HC, Tang J and Feng WH: MicroRNA-30c modulates type I IFN responses to facilitate porcine reproductive and respiratory syndrome virus infection by targeting JAK1. J Immunol 196: 2272-2282, 2016. 